\title{
Facile Synthesis of Hollow Carbon Nanospheres and Their Potential as Stable Anode Materials in Potassium Ion Batteries
}

\author{
Xian-Sen Tao ${ }^{\dagger, t}$, Yong-Gang Sun ${ }^{\dagger}$, Yuan Liu ${ }^{\dagger,}$, Bao-Bao Chang ${ }^{\S}$, Chun-Tai Liu ${ }^{\S}$,

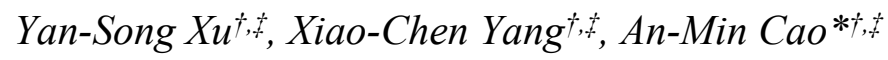

†CAS Key Laboratory of Molecular Nanostructure and Nanotechnology, and Beijing National Laboratory for Molecular Sciences, Institute of Chemistry, Chinese Academy of Sciences (CAS), Beijing 100190, People's Republic of China.

¥University of Chinese Academy of Sciences, Beijing 100049, People’s Republic of China.

$\S$ Key Laboratory of Materials Processing and Mold, Ministry of Education, National Engineering Research Center for Advanced Polymer Processing Technology, Zhengzhou University, Zhengzhou, 450002, China 

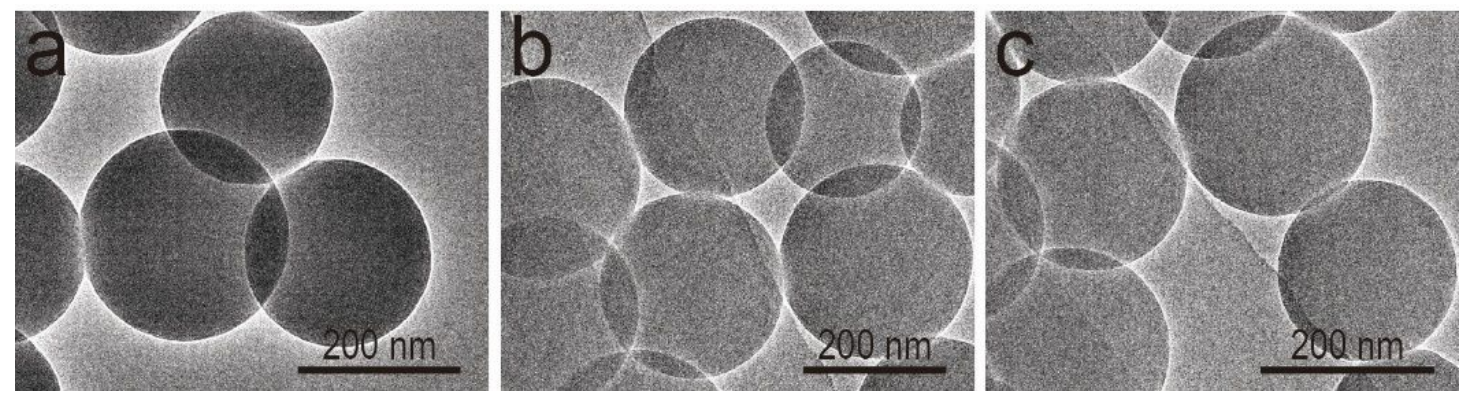

Figure S1. TEM images of the PmPD collected at different times (a) $20 \mathrm{~min}$ (b) $40 \mathrm{~min}$ (c) $90 \mathrm{~min}$. All the samples were washed by ethanol.
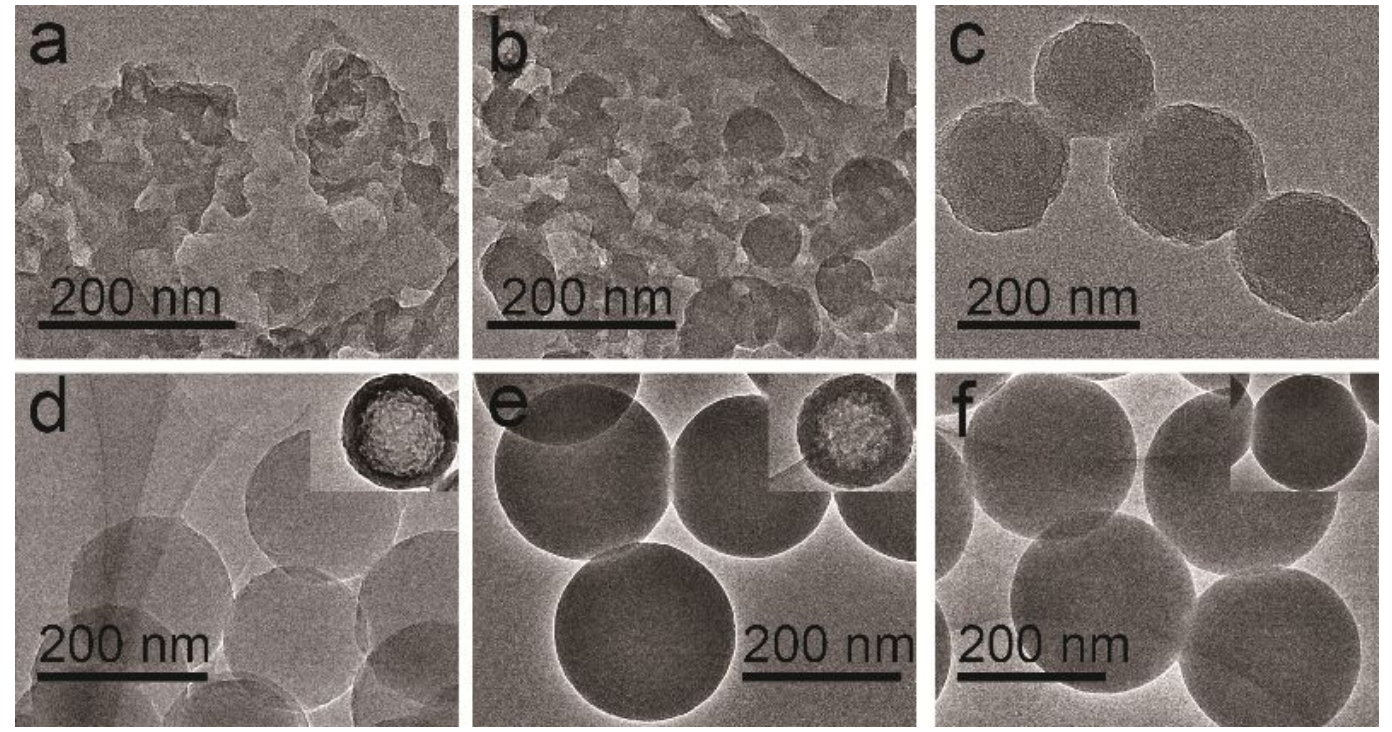

Figure S2. TEM images of the Cu-PmPD collected at different times (a) $30 \mathrm{sec}$ (b) $1 \mathrm{~min}$ (c) $3 \mathrm{~min}$ (d) $15 \mathrm{~min}$ (e) $30 \mathrm{~min}$ (f) $8 \mathrm{~h}$, the insets of (d) (e) (f) exhibit the morphology evolution of the samples treated by water.

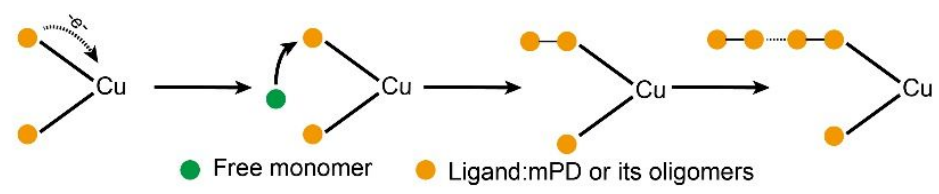

Figure S3. Possible mechanism for the formation of Cu-PmPD 

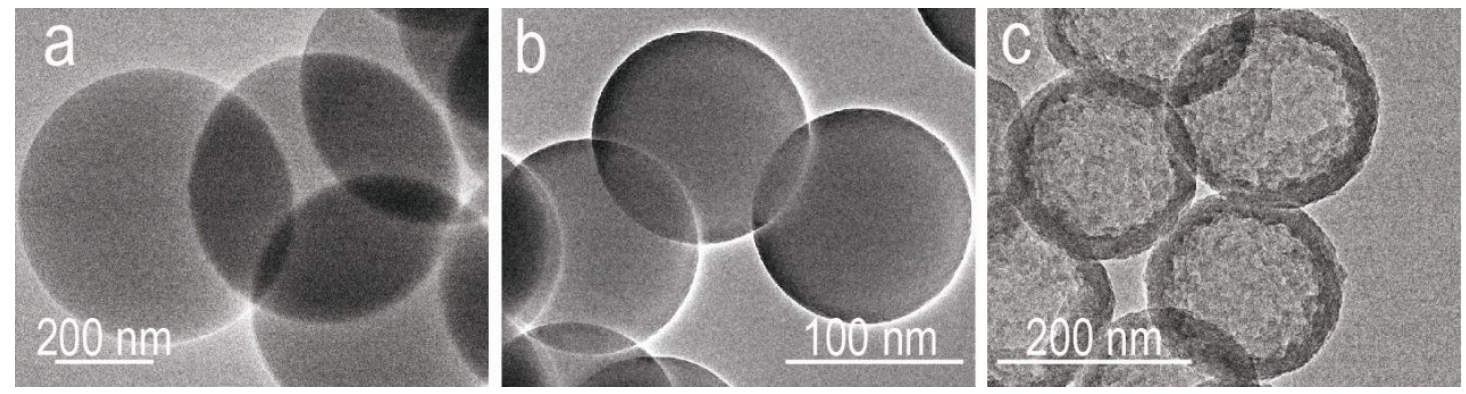

Figure S4. TEM images of Cu-PmPD (a) the reactant concentration was three times than Fig.1, (b) the reaction temperature was $60^{\circ} \mathrm{C}$, (c) the reaction temperature was $0^{\circ} \mathrm{C}$.

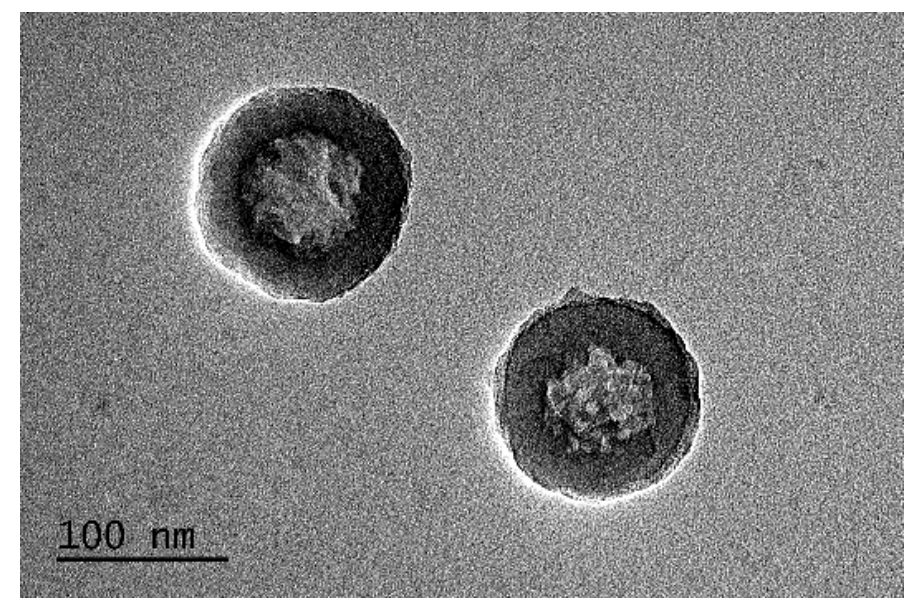

Figure S5 TEM image of the Cu-PmPD nanospheres with a size of 150nm
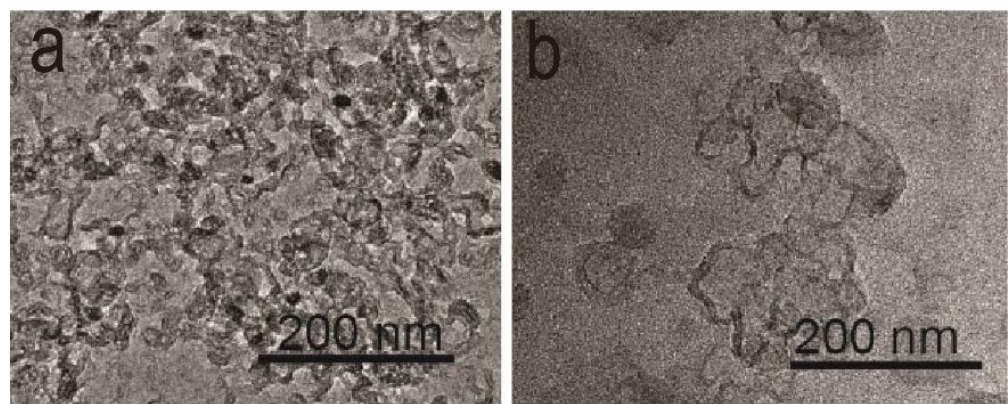

Figure S6. TEM images of the Cu-PmPD treated by different solvents (a) N, N-Dimethylformamide (DMF), (b) N- Methyl pyrrolidone (NMP). 

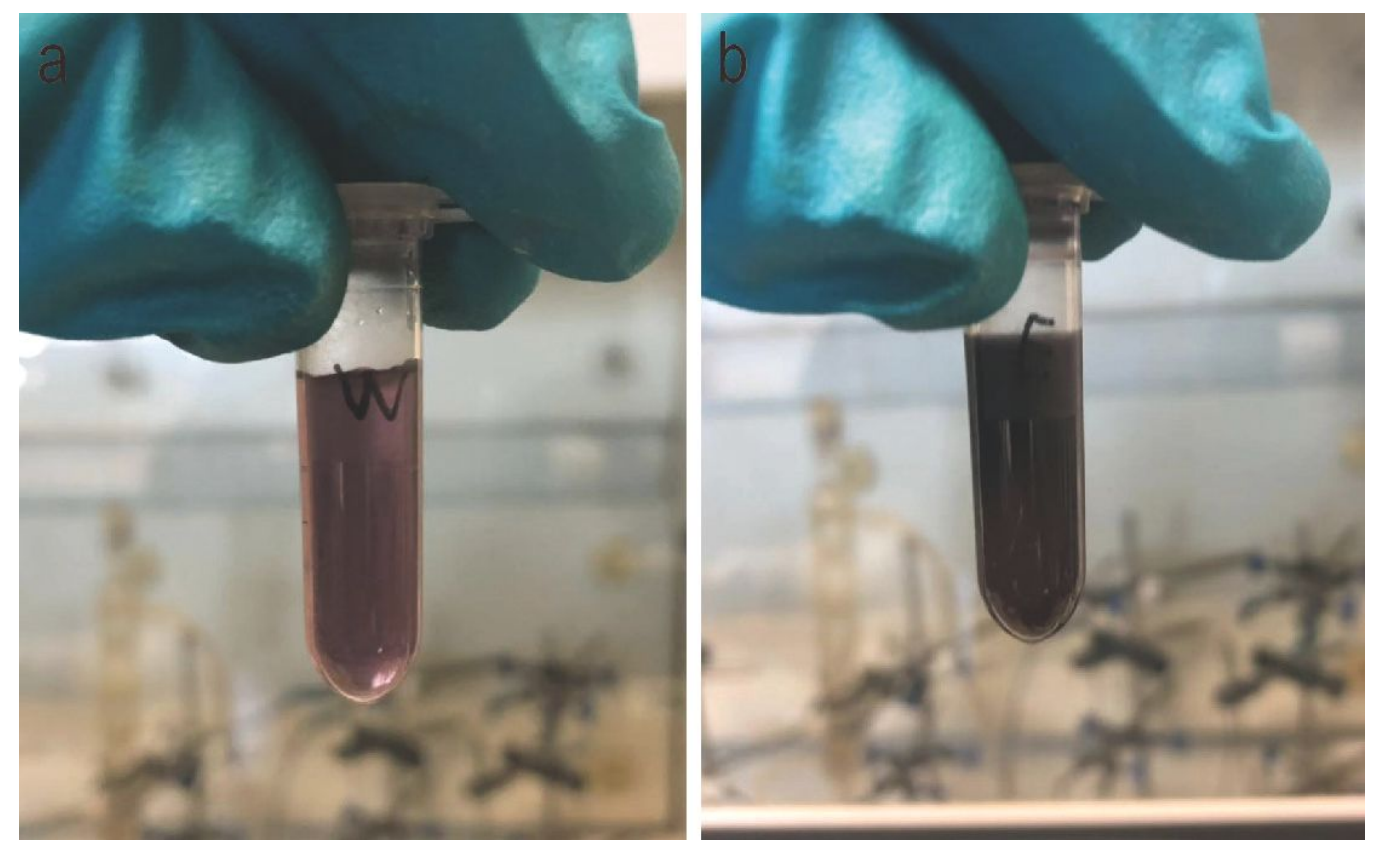

Figure S7. Experimental photos of the samples after treated by water (a) and ethanol (b). The samples were collected in $1 \mathrm{~min}$.

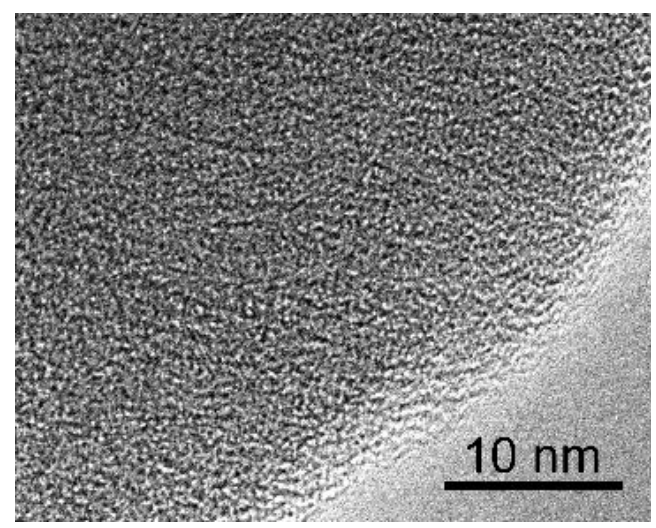

Figure S8. The HRTEM image of HCNs obtained at $1200^{\circ} \mathrm{C}$.

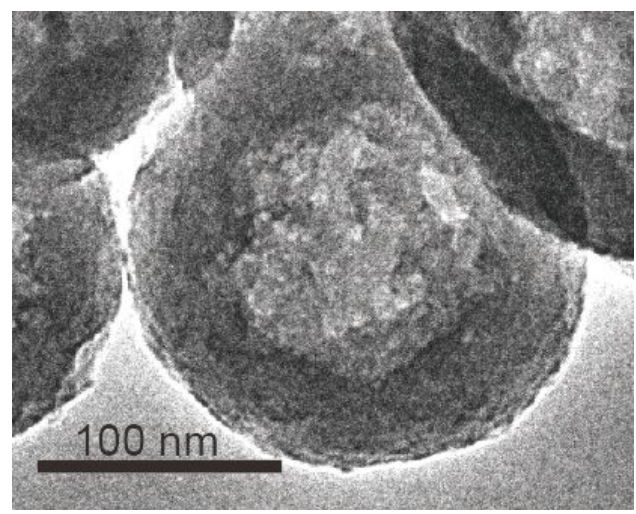

Figure S9. The TEM image of the HCNs after 100 electrochemical cycles. 